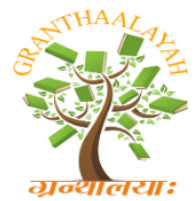

\author{
INTERNATIONAL JOURNAL OF RE
GRANTHAALAYAH \\ A knowledge Repository
}

Science

\title{
FOOD HABITS AND CULTURE FACTORS IN PREGNANT ADOLESCENTS
}

\author{
MSc. Cecilia Herrera Martinez ${ }^{1}$, Dr. Jorge Daher Nader ${ }^{1}$, MSc. Yesica Pazmiño Mera ${ }^{1}$, \\ MSc. Rocio Fonseca Tumbaco ${ }^{1}$, MSc. Vicky Narea Morales ${ }^{1}$ \\ ${ }^{1}$ School of Medicine, Faculty of Medical Sciences, University of Guayaquil, Ecuador
}

\begin{abstract}
Adequate eating habits during the gestation period of pregnant teenagers are of vital importance because this will affect the process of formation of the fetus at birth. If pregnant adolescents have an early pregnancy, the chances of a poor physiological maturity of the fetus are more likely to be executed, which makes it necessary to correctly implement the process of feeding during pregnancy, if possible eliminate all bad habit of feeding. The adolescent should seek the positive contribution of nutrients and food that contribute both to the fetus and the mother energy, which will be useful at birth. It should be noted that multiple studies in adolescent pregnant cultural factor food affects directly proportional to the time of feeding, in cases where the teenager is educated positively on their food is ingested foods balanced diet, eliminating all those foods that contain preservatives chemicals and processed foods that deteriorate the health of the pregnant woman. For the development of this research is to determine through the survey process the habits food and cultural factors of adolescent pregnant according to the sector, as well as also those relating theoretical to provide knowledge of the habits eating in adolescents pregnant, and also determine the nutrition of pregnant adolescents in relation to the gestational age of the patient according to the BMI, and identify the nutrition of pregnant women by developing an educational talk proposal in order to correct bad eating habits.
\end{abstract}

Keywords: Cultural Factors; Pregnancy; Teenagers; Food Habits; Nutrition.

Cite This Article: MSc. Cecilia Herrera Martinez, Dr. Jorge Daher Nader, MSc. Yesica Pazmiño Mera, MSc. Rocio Fonseca Tumbaco, and MSc. Vicky Narea Morales. (2020). "FOOD HABITS AND CULTURE FACTORS IN PREGNANT ADOLESCENTS." International Journal of Research - Granthaalayah, 8(2) 1-7. https://doi.org/10.29121/granthaalayah.v8.i2.2020.174.

\section{Introduction}

The habits eating in pregnancy of adolescents are a problem, because this directly affects the process of nutrition and direct impact on the process of formation of adolescent pregnant. All the studies carried out in the gestational development point to the correct handling of foods that contribute positively to the product of pregnancy, thereby avoiding health problems such as preclamsia, premature delivery, gestational diabetes. The maternity process is determined as a 
physiological function to which the best is conditioned to give rise to a baby according to certain conditions that are preceded by the pregnancy process, requiring the mother to have the necessary care and responsibility of the process of food intake during maternity. During the pregnancy process, multiple factors that affect the health of both the mother and the baby intervene, among them the incidence of hormones that will precede the health characteristics of individuals.

The habits eating in adolescent pregnant women rely heavily on factors external such as the relative psychological, socioeconomic and cultural influence directly on the health of the mother, if this is untidy in their power could lead to the chain of negative health consequences of the mother and the fetus in the process of formation, several statistical studies carried out show that the rate of adolescent pregnant women year after year is increased generating a phenomenon of early pregnancies, being necessary that they implement diets according to their gestational age rich in zinc, folic acid, magnesium, vitamin D, E, B6 above all calcium and iron essential elements for the process of formation of the fetus. it is necessary that during pregnancy there is awareness in the adolescent's habits food usually ingested modified, not only to satisfy the hunger of the expectant mother, but provide the necessary nutrients for developing gestational optimal. (15)

The process malnutrition in pregnant adolescents largely depends closely on the habits food running during the period of gestation. This could give as a result that the infant to be born underweight and get the boxes mother showing anemia, syndrome of hypertension identified during pregnancy. eating habits in pregnant teenagers depend largely on the diversity of the culture that this teenager has, the most evident mentioned during the studies carried out is because the family and the pregnant woman eat foods high in saturated fat, directly affecting the embryo in gestation presenting a picture of malnutrition both the pregnant teenager and the fetus ( 17 ). The incidence of habits food depends closely to sociocultural factors of patients because there is no efficient control of food, according to data obtained in studies most of the teenagers are driven by what in Latin America is known as cravings, which largely consist of treats, and fried foods that negatively affect the gestation process . Leaving aside the products rich in proteins, whole grains, vegetables and fruits that provide vitamins, and iron that are necessary during this period, which requires pregnant women to have a rich, healthy and balanced diet for their body.

The general objective is to determine the eating habits, as well as the cultural factors in pregnant adolescents attending the Francisco Jácome Health Center, being able to elaborate the determination and relationship existing in eating habits of pregnant adolescents with their chronological age. Further they developed so specific determination habits and nutritional factors related to nutrition of pregnant adolescents relating gestational age of the patients through their BMI; the nutrition of adolescent is pregnant related to gestational age according to their BMI.

As a critical question, the possibility exists in determining the existence of a relationship between the nutritional level of pregnant adolescents according to their BMI and their chronological age.

\section{To Pregnant Teenagers}

According to data from studies carried out the feeding of pregnant women are bound by the conditions socioeconomic adolescent, the same as largely are poor and in some cases lack the support economic her parents what causes sexually begin early lack of education sexual and unaware of methods of prevention and family control, obtained as a result of early pregnancies are 
often in countries of Latin America. Early pregnancy directly affects the health of the pregnant woman and the fetus, which causes high rates of both maternal and perinatal morbidity and mortality. Poor feeding could cause the pregnant woman to suffer from gestational diabetes, preclamsia, hypertension and, above all, benefit the fetus with a diet rich in vitamins, proteins, carbohydrates and minerals necessary for the process of pregnancy formation, at this time both the mother and the Product fight for the assimilation of resources. (16)

Habit food in adolescents pregnant HAB correct it food in teenage pregnancy in the international context is of vital importance and it should be taken as a mainstream problem on to due to lack of nutritional culture combined with the fact of scarce resources economic that the pregnant adolescent can have, and above all it is framed in the social context because the adolescents are closely influenced by the family group at the time of feeding, consuming foods that do not provide both the mother and the fetus with nutrients necessary for gestational development. to the existence incorrect intake of foods with nutrients crescents will result in weight and health of the mother and fetus are wrong, therefore it is vital to be made on time due dietary control during pregnancy which They should be a diet rich in iron, calcium and, in the absence of sugar and fat, which will automatically have an impact on gestational diabetes, overweight or obesity by framing these pregnant adolescents as high-risk pregnancies. Family food culture is essential in this period of pregnancy, in short, bad eating habits are equal to adverse maternal effects and negative effects on the fetus, low birth weight, lower postnatal growth to the established parameters. (21)

The cultural factors involved in the field of habits food of adolescent pregnancy and the cultural factor according to studies are closely linked to the development everyday teen, who's unaware of the proper way to feed itself that, in the household only ingest junk food and processed food with high chemical content that the adolescent who is currently in the process of pregnancy would not provide necessary nutrients. whichever implemented in the family group the reduction of intake of sausages or meats with saturated fat in amounts only cause the elevation of cholesterol and triglycerides in the blood. (1)

\section{Materials and Methods}

The methodology for development and valuation on the nutritional status of pregnant teenagers proceeded to execute the identification of BMI for each patient during the week tenth, calculating the weight as the gestating increase the same and height, using the standard BMI formula ( Body Mass Index), with the pregnant woman being represented in kilograms over the size (height) in squared meters, and the patient's BMI will be recorded in each prenatal evaluation using that the graph $\mathrm{f}$ ICOS relevant developed with the nomogram tool, with which you can perform two dimensional calculation based on any variable; It noting that the increase to the weight of the patient and allowing to make a projection of the weight estimated for the next month, the formula is defined standard of the BMI below :

$\mathrm{BMI}=(\mathrm{kg}) /(\mathrm{m})^{\wedge} 2$

The number of adolescents defined for this study is determined by 50 adolescents, who will be treated for the purpose of the present study at the Francisco Jácome health center, the ages implemented in the research range from 11 years to 20 years old, for this purpose was used as a 
model statistical the analysis of chi square, with the same which may identify the existence of differences hypothesis null and hypotheses positive in research, interacting in the study is $t$ nutritional ado in relation at the age of pregnant teenage patients.

Table 1: Adolescents according to age.

\begin{tabular}{|c|c|}
\hline Age & Teenagers \\
\hline 14 & 1 \\
\hline 15 & 2 \\
\hline 16 & 5 \\
\hline 17 & 13 \\
\hline 18 & 15 \\
\hline 19 & 14 \\
\hline Total & 50 \\
\hline
\end{tabular}

\section{Results and Discussions}

In the development of the research, a survey was conducted aimed at identifying the nutritional status of pregnant women, correlating this with their gestational age and BMI, in the study the groups of adolescents were divided according to their age, identifying the individual in the which nutritional status is mostly affected in adolescence.

Table 2: Age table for pregnant adolescents according to their nutritional status

\begin{tabular}{|c|c|c|c|c|c|c|c|}
\hline Age & Teenagers & Low & Normal & Overweight & \multicolumn{3}{|c|}{ Obesity } \\
\cline { 6 - 8 } & & & & & OG1 & OG2 & OG3 \\
\hline 14 & 1 & 0 & 1 & 0 & 0 & 0 & 0 \\
\hline 15 & 2 & 0 & 1 & 1 & 0 & 0 & 0 \\
\hline 16 & 5 & 1 & 3 & 0 & 1 & 0 & 0 \\
\hline 17 & 13 & 0 & 5 & 1 & 6 & 0 & 0 \\
\hline 18 & 15 & 0 & 2 & 3 & 9 & 2 & 0 \\
\hline 19 & 14 & 0 & 1 & 5 & 5 & 3 & 0 \\
\hline Total & 50 & 1 & 13 & 10 & 21 & 5 & 0 \\
\hline
\end{tabular}

Through the value of the BMI, it was possible to identify that pregnant adolescents have a high tendency to grade 1 obesity as there are $42 \%$ of pregnant adolescents with this problem, the same as those at the age of 18 have the highest incidence of this nutritional condition, while $23 \%$ of adolescents are in the normal values of nutrition, a value close to half of the cases with obesity grade 1, being those who are 17 years old who take care of their level of overweight.

Table 3: Summary of data for the Chi-square Test

\begin{tabular}{|l|c|c|c|c|c|c|}
\hline \multirow{2}{*}{} & \multicolumn{5}{|c|}{ Cases } \\
\cline { 2 - 7 } & \multicolumn{2}{|c|}{ Valid } & \multicolumn{2}{c|}{ Lost } & \multicolumn{2}{c|}{ Total } \\
\cline { 2 - 7 } & $\mathbf{N}$ & Percentage & N & Percentage & N & Percentage \\
\hline Age * NUTRITIONAL & fifty & $100.00 \%$ & 0 & $0.00 \%$ & fifty & $100.00 \%$ \\
\hline
\end{tabular}


Table 4: Data table for Chi square test

\begin{tabular}{|c|c|c|c|c|c|c|}
\hline & \multicolumn{7}{|c|}{ Nutritional } \\
\hline Age & Low & Normal & Overweight & OG1 & OG2 & Total \\
\hline \multirow{2}{*}{14} & 0 & one & 0 & 0 & 0 & one \\
\cline { 2 - 7 } & $0.00 \%$ & $9.09 \%$ & $0.00 \%$ & $0.00 \%$ & $0.00 \%$ & $2.00 \%$ \\
\hline \multirow{2}{*}{ fifteen } & 0 & one & one & 0 & 0 & two \\
\cline { 2 - 7 } & $0.00 \%$ & $9.09 \%$ & $9.09 \%$ & $0.00 \%$ & $0.00 \%$ & $4.00 \%$ \\
\hline \multirow{2}{*}{16} & one & 3 & 0 & one & 0 & 5 \\
\cline { 2 - 7 } & $100.00 \%$ & $27.27 \%$ & $0.00 \%$ & $4.55 \%$ & $0.00 \%$ & $10.00 \%$ \\
\hline \multirow{2}{*}{17} & 0 & 4 & two & 7 & 0 & 13 \\
\cline { 2 - 7 } & $0.00 \%$ & $36.36 \%$ & $18.18 \%$ & $31.82 \%$ & $0.00 \%$ & $26.00 \%$ \\
\hline \multirow{2}{*}{18} & 0 & one & 3 & 9 & two & fifteen \\
\cline { 2 - 7 } & $0.00 \%$ & $9.09 \%$ & $27.27 \%$ & $40.91 \%$ & $40.00 \%$ & $30.00 \%$ \\
\hline \multirow{2}{*}{19} & 0 & one & 5 & 5 & 3 & 14 \\
\cline { 2 - 7 } & $0.00 \%$ & $9.09 \%$ & $45.45 \%$ & $22.73 \%$ & $60.00 \%$ & $28.00 \%$ \\
\hline \multirow{2}{*}{ Total } & one & eleven & eleven & 22 & 5 & fifty \\
\cline { 2 - 7 } & $100.00 \%$ & $100.00 \%$ & $100.00 \%$ & $100.00 \%$ & $100.00 \%$ & $100.00 \%$ \\
\hline
\end{tabular}

By identifying the data in the results we obtain that the differences are very little distanced by identifying that for each age range the values are little varied, even when there are significant differences between the different nutritional states, which is due to the fractionation of the adolescents according to their age range when there is only a 14 year old teenager when there are 1518 year old teenagers within the study.

Table 5: Hypothesis test for Chi square test

\begin{tabular}{|l|c|c|c|}
\hline Statistical & Value & df & Sig. Asint. (2-tails) \\
\hline Pearson's Chi-square & 30.18 & 20 & 0.067 \\
\hline Similarity Reason & 28.3 & 20 & 0.103 \\
\hline N of valid cases & 50 & & \\
\hline
\end{tabular}

When analyzing the results, we observe that there are differences in reference to nutritional status with age for the null hypothesis since the significance in the Pearson value exceeds 0.05; By obtaining this value we can deduce that the difference is not significant between nutritional status and age for pregnant adolescents.

\section{Conclusions and Recommendations}

As a result of the execution of the present investigation, it was possible to observe that pregnant adolescents of the medical center presented a high degree 1 obesity trend, which during pregnancy presented obesity conditions that could put the product's life at risk, which gives As a result, the nutritional status was established in obesity grade 1 and even grade 2, which is supposed to be guided by the ignorance of foods that contribute to nutrition during pregnancy. the same that can get corrected their runs early check pregnant plans or educational talks that cause them the awareness of the effects and consequences of poor nutritional status and impact that lead during and after the expected product. 
It is recommended to the study carried out to carry out a process of culture change in terms of the food that they eat as pregnant women, for this purpose, educational plans must be implemented that will make it possible to make known the gaps and corrective measures to the nutritional status present in pregnant women in turn correcting eating habits. In turn, simple food recipes must be implemented that provide the necessary nutrients in turn aimed at patients with food found in their environment, and that this is consistent with the needs required by each gestational stage of the adolescent and allows her to obtain a Good product and at the same time not neglecting their nutrition as this is an individual who is still in the final stage of personal development as a biological individual.

\section{Appendices}

Table 6: Weight gain assessment during pregnancy

\begin{tabular}{|l|l|}
\hline \multicolumn{2}{|c|}{ Valuation of Weight Gain During Pregnancy } \\
\hline Weight / Stature Category & Total Gain Recommended (Kg. \\
\hline Under BMI <19.8 & $12.5-18$ \\
\hline Normal BMI 19.8 to 26.0 & $11.5-16.0$ \\
\hline BMI overweight $>26-29$ & $7.0-11.5$ \\
\hline BMI Obesity $>29.0$ & 6.0 \\
\hline
\end{tabular}

\section{References}

[1] Acosta, V., \& Cárdenas, A. (2012). Pregnancy in adolescent sociocultural factors. Medical Magazine of the Mexican Social Security Institute.

[2] Apaza, D., \& Vildoso, M. (2012). Incidence and risk factors of low birth weight in population treated in hospitals of the Ministry of Health of Peru. Mexico.

[3] Constitutional Assembly. (2008). Constitution of the Republic of Ecuador. Ecuador: Official Registry.

[4] Behar, R., Gonzalez, J., \& Aguirre, A. (2010). Eating disorders in teenage pregnant women controlled in primary care. Re vista Chile Obstetrics Ginecol .

[5] Blasquez, M., Sobeida, M., Torres, F., \& Gogoeascoecha, T. (2010). Lifestyles in pregnant teenagers. Tabasco health magazine.

[6] Cantero, S. (2010). Morbid adolescent girls. Cuban Magazine Obstet Ginecol.

[7] National Congress. (1994). Free maternity law. Ecuador: Official Registry 523.

[8] De Benoist, BM (2012). Nutrition of women in the pregestational period, during pregnancy and during breastfeeding. WHO.

[9] Dominguez, A., \& Herazo, Y. (2010). Age of the adolescent pregnant woman as a risk factor for complications in pregnancy. Colombian Journal of Gynecology and Obstetrics.

[10] Donoso, B., \& Oyarzún, E. (2012). High risk pregnancy Atlanta

[11] Furness, P., McSeventy, A., \& Garland, C. (2011). Maternal Obesity support services a qualite study of the prespesctives of women and midwiwes . Pregnancy Childbirth

[12] Gónzalez, J., Schmidt, R., García, L., \& García, G. (2013). Analysis of food intake and nutritional habits in a population of adolescents in the city of Granada. Hospital Nutrition Magazine.

[13] Grandia, C., \& López, F. (2010). Gestational age estimation: Literature review. Redalyc.

[14] Guzmán, M., Vásquez, G., Troyo, R., \& Velarde, R. (2012). Food habits in Mexican pregnant adolescents according to their civil status. Hospital Nutrition Magazine.

[15] Herrera, S., Vásquez, G., Velarde, H., \& Romo, A. (2010). Feeding habits and cultural factors in pregnant adolescents. Magazine Latin American Archives of Nutrition. 
[16] León, P., Minassian, M., Burgundy, R., \& Bustamante, I. (2013). Teenage pregnancy. Electronic Pediatrics Magazine.

[17] León, P., Minassin, M., Burgundy, R., \& Bustamante, F. (2011). Teenage pregnancy. Electronic Pediatrics Magazine.

[18] López, C., \& Restrepo, M. (2014). A group of pregnant teenagers perceptions regarding their pregnancy accompanied by food insecurity. Biblical Health Magazine.

[19] López, C., Restrepo, M., \& Salas, L. (2014). Gestation amid food insecurity; perceptions of a group of pregnant teenagers. Public health magazine.

[20] Medeiros, M., Bacarau, P., \& Vieria, C. (2015). Dietary patters of young adolescents in urban areas of Northeast Brazil. Hospital Nutrition.

[21] Motta, S., Oliveira, Q., Monteriro, B., \& Oliveira, A. (2015). Food consumption patters during pregnancy a longitudinal study in a region of the north east of Brazil. Hospital Nutrition.

[22] Muñoz, N., Martínez, W., \& Quintero, A. (2010). Validation of the Latin American and Caribbean food security scale in pregnant teenagers. Public Health Magazine.

[23] Noguera, O., \& Alvarado, H. (2011). Teen pregnancy: A look from nursing care. Clinical Nursing Magazine.

[24] Official Mexican Standard. (2013). Promotion and education for health in alimentary matters. Criteria to provide guidance. Mexico: Official Mexican Standard.

[25] WHO. (2011). Teen pregnancy: A culturally complex problem. Bulletin of the Organization World of Health.

[26] Pérez, F., Bastardo, E., Cermeño. Y. , \& Díaz , L. (2010). Incidence of early pregnancy according to the stages of adolescence. Informed

[27] Rangel, D., González, R., Barrera, H., \& Pereda, C. (2012). Pregnancy in adolescense: Its behavior in San Luis municipality. Medical Sciences Magazine.

[28] Restrepo, M., \& Manjarrés, M. (2013). Nutritional status and eating habits in indigenous adolescents embera of Colombia. Rev. Chile Nutrition.

[29] Samano, S., Godinez, M., \& Romero, P. (2014). The socio - cultural and eatings aspects of women experiencing risk pregnacies. Science and collective health magazine.

[30] Sánchez, J., Sámano, S., \& Pinzón, R. (2013). Sociocultural and environmental factors that influence food and environmental practices that influence the nutritional practices of pregnant women with and without obesity. Cuba.

*Corresponding author.

E-mail address: ceci-mar3@ hotmail.com/jdaher_nader@yahoo.es/ jessicapaz1980@ hotmail.com/ rocio.fonsecat@ug.edu.ec/vicky.naream@ug.edu.ec 